\title{
Activated Cdc42-associated kinase Ack1 promotes prostate cancer progression via androgen receptor tyrosine phosphorylation
}

Nupam P. Mahajan*†, Yuanbo Liu*, Samarpan Majumder*, Maria R. Warren ${ }^{\ddagger}$, Carol E. Parker, James L. Mohler*§, H. Shelton Earp* ${ }^{* \uparrow \|}$, and Young E. Whang*१||**

*Lineberger Comprehensive Cancer Center, Departments of ${ }^{+}$Pharmacology, ${ }^{\ddagger}$ Biochemistry and Biophysics, ${ }^{9}$ Medicine, and **Pathology, University of North Carolina at Chapel Hill, Chapel Hill, NC 27599; and §Department of Urologic Oncology, Roswell Park Cancer Institute, Buffalo, NY 14263

Edited by Owen N. Witte, University of California, Los Angeles, CA, and approved March 29, 2007 (received for review January 16, 2007)

Activation of the androgen receptor (AR) may play a role in androgenindependent progression of prostate cancer. Multiple mechanisms of AR activation, including stimulation by tyrosine kinases, have been postulated. We and others have recently shown involvement of activated Cdc42-associated tyrosine kinase Ack1 in advanced human prostate cancer. Here we provide the molecular basis for interplay between Ack1 and AR in prostate cancer cells. Activated Ack1 promoted androgen-independent growth of LNCaP and LAPC-4 prostate xenograft tumors, AR recruitment to the androgen-responsive enhancer, and androgen-inducible gene expression in the absence of androgen. Heregulin-stimulated HER2 activation induced Ack1 activation and AR tyrosine phosphorylation. Ack1 knockdown inhibited heregulin-dependent AR tyrosine phosphorylation, AR reporter activity, androgen-stimulated gene expression, and AR recruitment. Ack1 was recruited to the androgen-responsive enhancers after androgen and heregulin stimulation. In 8 of 18 primary androgenindependent prostate tumor samples, tyrosine-phosphorylated AR protein was detected and correlated with the detection of tyrosinephosphorylated Ack1. Neither was elevated in androgen-dependent tumors or benign prostate samples. Activated Ack1 phosphorylated AR protein at Tyr-267 and Tyr-363, both located within the transactivation domain. Mutation of Tyr-267 completely abrogated and mutation of Tyr-363 reduced Ack1-induced AR reporter activation and recruitment of $A R$ to the androgen-responsive enhancer. Expression of AR point mutants inhibited Ack1-driven xenograft tumor growth. Thus, Ack1 activated by surface signals or oncogenic mechanisms may directly enhance AR transcriptional function and promote androgenindependent progression of prostate cancer. Targeting the Ack1 kinase may be a potential therapeutic strategy in prostate cancer.

Ack1 tyrosine kinase | signal transduction | HER2 | cross-talk

nevitable progression of prostate cancer to the hormone refractory stage makes prostate cancer the second leading cause of cancer deaths in American men. Although this is referred to as androgen-independent prostate cancer (AICaP), the androgen receptor (AR) is likely to be involved in the recurrence of most prostate cancers. Mechanisms of AR activation in AICaP include AR gene amplification or mutation, overexpression of AR or coactivators, and cross-talk with tyrosine kinases such as HER2 (ErbB2) $(1,2)$. Although tyrosine kinases have been implicated in prostate cancer $(3,4)$, mechanisms by which tyrosine kinases contribute to the pathogenesis and progression of prostate cancer have not been completely elucidated.

We and others have recently shown involvement of activated Cdc42-associated tyrosine kinase Ack1 in advanced human prostate cancer $(5,6)$. Ack1 was identified as a $120-\mathrm{kDa}$ protein that interacts with activated GTP-bound Cdc42. Structural studies have revealed sites of Cdc42 and Ack1 interaction and an autoinhibitory mode of kinase regulation (7). The latter can be abrogated by a single-point mutation L487F, yielding a constitutively activated Ack1 kinase $(5,8)$. Expression of this activated form of Ack1 markedly accelerated prostate xenograft tumor growth in mice (5). The tumorigenic role of activated Ack1 in prostate cancer was further supported by increased levels of tyrosine-phosphorylated Ack1 found in clinical specimens of AICaP (5). Ack1 amplification and overexpression occurred in many tumor types (including prostate), was correlated with poor prognosis, and was associated with increased cell motility and invasiveness in vitro and metastasis in vivo $(6,9)$. Knockdown of Ack1 increased apoptosis in transformed cells, suggesting that Ack1 signaling enhanced survival $(10,11)$. Ack1 also modulated the level of a putative tumor suppressor, Wwox, by targeting it for polyubiquitination and proteasome-mediated destruction (5). Taken together, these recent data suggest that Ack1 signaling may contribute in multiple ways to tumorigenesis. In this report, we provide evidence for an Ack1-dependent process, site-specific tyrosine phosphorylation of AR, promoting ARregulated activities in a low-androgen environment.

\section{Results}

Activated Ack1 Promotes Androgen-Independent Growth of Prostate Xenograft Tumors, Androgen-Regulated Gene Expression, and AR Recruitment. The effect of activated Ack1 kinase on tumor formation was tested in castrated mice. Vector control LNCaP cells did not form tumors in castrated mice (Fig. $1 A$ ), consistent with previous reports that LNCaP cells are androgen-dependent for tumorigenicity $(1,12)$. However, constitutively activated caAck-expressing LNCaP cells formed tumors rapidly in castrated mice (Fig. $1 A$ ). In additional experiments, half of the mice injected with LNCaP-caAck cells were castrated at day 14; subsequent tumor growth was unaffected by castration [supporting information (SI) Fig. 7]. An additional model, LAPC-4 prostate cancer cells expressing caAck vs. kinase dead kdAck, was tested. caAck-expressing LAPC-4 cells formed tumors in castrated mice, although more slowly than LNCaP-caAck, whereas kdAck-expressing LAPC-4 cells did not form tumors (Fig. 1B).

Author contributions: H.S.E. and Y.E.W. contributed equally to this work; N.P.M., H.S.E., and Y.E.W. designed research; N.P.M., Y.L., S.M., M.R.W., and C.E.P. performed research N.P.M., M.R.W., C.E.P., and J.L.M. contributed new reagents/analytic tools; N.P.M., Y.L., M.R.W., C.E.P., J.L.M., H.S.E., and Y.E.W. analyzed data; and N.P.M., J.L.M., H.S.E., and Y.E.W. wrote the paper.

The authors declare no conflict of interest.

This article is a PNAS Direct Submission.

Abbreviations: $A D C a P$, androgen-dependent prostate cancer; $\mathrm{AICaP}$, androgen-independent prostate cancer; $A R$, androgen receptor; ARE, androgen response element; $B P H$ benign prostate hyperplasia; DHT, dihydrotestosterone; hk2, human kallikrein 2; PSA, prostate-specific antigen.

|To whom correspondence may be addressed. E-mail: hse@med.unc.edu or ywhang@ med.unc.edu.

This article contains supporting information online at www.pnas.org/cgi/content/full/ 0700420104/DC1.

() 2007 by The National Academy of Sciences of the USA 

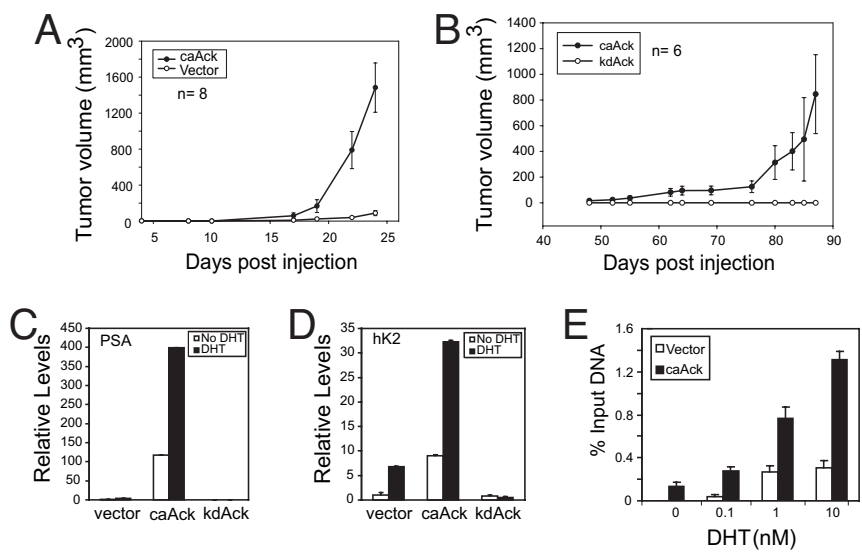

Fig. 1. Activated Ack1 promotes androgen-independent growth of prostate xenograft tumors, androgen-regulated gene expression, and AR recruitment. (A) LNCaP cells ( $2 \times 10^{6}$ cells per injection) stably expressing caAck or vector control were injected s.c. into the flanks of castrated nude mice. (B) LAPC-4 cells $\left(2 \times 10^{6}\right.$ cells per injection) stably expressing caAck or kinase dead kdAck were injected s.c. into the flanks of castrated nude mice. ( $C$ and $D$ ) LNCaP cells stably expressing caAck, kdAck, or vector were treated with dihydrotestosterone (DHT) (10 nM) for $12 \mathrm{~h}$. Quantitative RT-PCR for PSA (C) and hK2 (D) mRNA was performed. Data shown are representative of three similar independent experiments. $(E)$ LNCaP cells stably expressing caAck or vector were treated with DHT for $2 \mathrm{~h}$, and ChIP analysis for AR binding to the androgen response element (ARE) III enhancer of the PSA gene was performed by using quantitative PCR. Data shown are representative of three similar independent experiments.

To test whether Ack1-mediated androgen independence extended to gene expression, mRNA for two AR-regulated genes, prostate-specific antigen (PSA) and human kallikrein 2 (hK2), was measured. PSA and hK2 mRNA levels were higher in androgen-deprived caAck-expressing LNCaP (Fig. $1 C$ and $D$ ) and LAPC-4 cells (SI Fig. 7) than in androgen-stimulated vector control or kdAck-expressing LNCaP and LAPC-4 cells. Addition of androgen to caAck-expressing cells further stimulated PSA and hK2 mRNA levels (Fig. $1 C$ and $D$ and SI Fig. 7), indicating that AR-mediated transcription occurred in the absence of androgen in caAck-expressing cells and was induced to a higher level on androgen stimulation. Knockdown of AR by RNA interference in caAck-expressing LNCaP cells led to substantial inhibition of both basal and androgen-stimulated PSA and hK2 expression, demonstrating that increased PSA and hK2 expression by activated Ack1 required AR (SI Fig. 8). To assess potential mechanisms of enhanced AR-dependent transcription, chromatin immunoprecipitation (ChIP) analysis of the PSA enhancer was performed. In caAck-expressing cells, more AR protein was bound to the androgen-responsive enhancer in the absence or at suboptimal concentrations of androgen compared with vector control cells (Fig. $1 E$ ). In contrast, a non-AR target gene p16 did not exhibit AR binding to its promoter either in vector or caAck cells (data not shown). Taken together, these data showed that sustained Ack1 activity resulted in tumor growth in the absence of testicular androgens and in hyperresponsiveness of AR-dependent transcription to low levels of androgen.

Activated Ack1 Binds and Phosphorylates AR at Tyrosine Residues. To identify the mechanisms of AR-regulated transcriptional activation by Ack1, interaction between Ack1 and AR was examined. LNCaP and LAPC-4 cells expressing myc-tagged Ack1 constructs (5) were immunoprecipitated with anti-myc antibody, followed by immunoblotting with anti-AR antibody. caAck, but not kdAck, was found in complex with endogenous AR protein (Fig. $2 A$ and $B$, lane 3 ), suggesting that the Ack1-AR complex
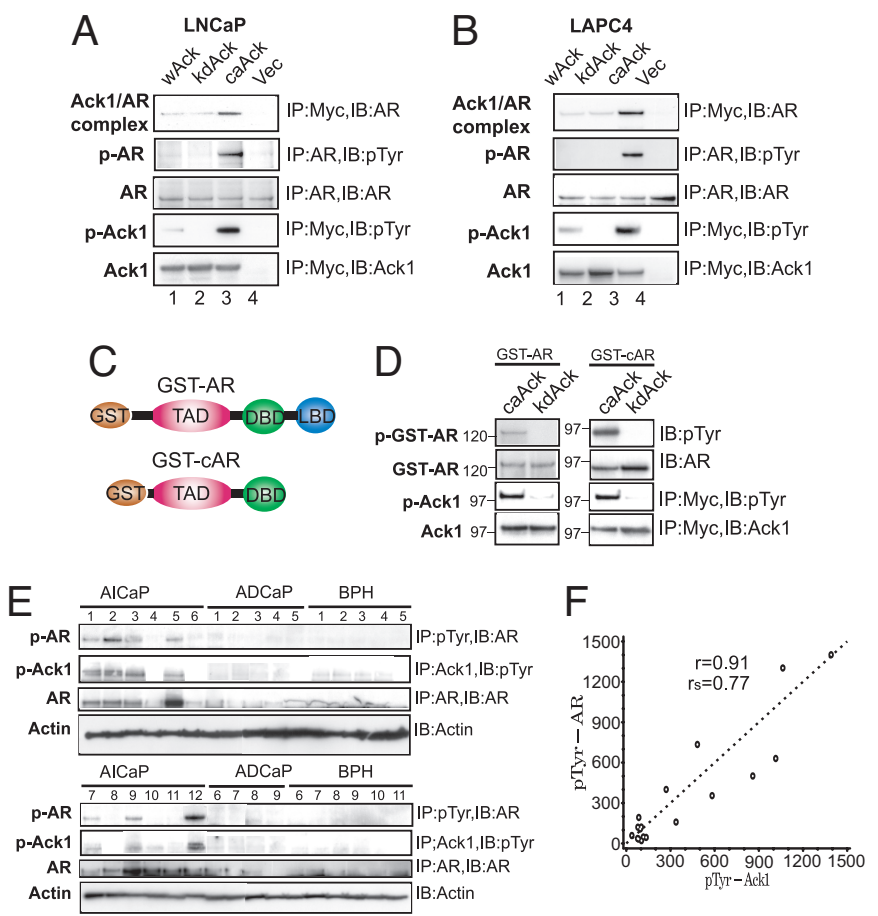

Fig. 2. Activated Ack1 binds and tyrosine phosphorylates $A R$ in vivo and in vitro; primary human androgen-independent prostate cancer specimens express tyrosine-phosphorylated AR and Ack1. ( $A$ and $B$ ) LNCaP or LAPC-4 cells expressing myc-tagged Ack1 constructs were immunoprecipitated by using antibodies described, followed by immunoblotting analysis. (C) Schematic of GST-AR and GST-CAR constructs. TAD, transactivation domain; DBD, DNAbinding domain; LBD, ligand-binding domain. (D) Purified GST-AR and GSTCAR proteins were incubated with caAck or kdAck and ATP. Ack and GST-AR proteins were separated, electrophoresed, and subjected to immunoblotting analysis. $(E)$ Equal amounts of protein from lysates of AICaP, ADCaP, and BPH samples were subjected to immunoprecipitation, followed by immunoblotting as indicated. $(F)$ The levels of tyrosine-phosphorylated AR and Ack 1 from immunoblots of AICaP samples were quantified by using SCION Image (Frederick, MD) software and plotted in arbitrary units. Pearson's product-moment correlation coefficient $(r)$ and Spearman's rank correlation coefficient $\left(r_{\mathrm{s}}\right)$ were calculated by using SAS statistical software, version 9.1 (SAS Institute, Cary, NC).

formation was dependent on Ack1 kinase activity. In cells expressing caAck, but not in other cells, endogenous AR was tyrosine phosphorylated (Fig. $2 A$ and $B$, lane 3 ). To determine whether AR is a direct substrate of Ack1 kinase, purified GST-AR fusion proteins (Fig. $2 C$ ) were incubated with immunopurified caAck or kdAck in an in vitro kinase reaction. caAck, but not kdAck, tyrosine phosphorylated both GST-AR and GST-cAR (lacking the ligand-binding domain) (Fig. 2D), demonstrating that Ack1 directly phosphorylated AR in the $\mathrm{N}$ terminal region of AR.

AR and Ack1 Proteins Are Tyrosine Phosphorylated in Primary AndrogenIndependent Prostate Tumors. Previously, we reported that primary AICaP tumors, but not benign prostate hyperplasia (BPH), had approximately a 5-fold increase in tyrosine-phosphorylated Ack1 (5). Because activated Ack1 enhanced androgenindependent xenograft tumor growth, AR-regulated gene expression, and AR tyrosine phosphorylation in prostate cancer cell lines, we assessed tyrosine phosphorylation of AR in 49 different human prostate samples (Fig. 2E, SI Fig. 9, and data not shown). In 8 of 18 AICaP tumors $(\approx 44 \%)$, tyrosinephosphorylated AR was detected. However, none of 13 androgen-dependent prostate cancer (ADCaP) tumors or $18 \mathrm{BPH}$ 
samples exhibited tyrosine-phosphorylated AR (Fig. 2E). The total amount of AR was higher in AICaP samples, an observation consistent with earlier work (1). Those AICaP tumors that exhibited higher levels of tyrosine-phosphorylated AR also expressed higher levels of tyrosine-phosphorylated Ack1 (e.g., tumors $1-3,5,7,9$, and 12, Fig. 2E). A strong correlation between tyrosine-phosphorylated AR and Ack1 in AICaP tumor tissue was demonstrated by a Pearson's product-moment correlation coefficient of 0.91 and a Spearman's rank correlation coefficient of 0.77 (Fig. 2F). Xenograft tumors expressing activated Ack1 that were harvested from castrated mice also expressed tyrosine-phosphorylated AR (data not shown).

Heregulin-Mediated HER2 Tyrosine Kinase Activation Leads to Ack1 Activation and AR Phosphorylation. We and others have demonstrated that heregulin-dependent HER2/HER3 receptor tyrosine kinase signaling enhances AR activity (12-15); the effect of activated Ack1 on AR-dependent gene expression was similar to that which we observed by heregulin-dependent activation of the HER2/HER3 heterodimer (13). Therefore, we tested whether Ack1 may act as a downstream mediator of HER2/ HER3 on AR. Heregulin treatment of both LNCaP and LAPC-4 prostate cancer cells resulted in Ack1 tyrosine phosphorylation (Fig. $3 A$ ). To address the role of HER2 in heregulin-dependent Ack1 activation, we used LNCaP-scFv-5R cells, in which HER2 signaling was abolished by expression of a single-chain intracellular antibody against HER2 that sequesters nascent HER2 in the endoplasmic reticulum (14). Heregulin-induced activation of Ack1 was abolished in LNCaP-scFv-5R cells (Fig. 3B). These results suggested that HER2 stimulation activated Ack1. Next, we examined whether heregulin treatment of prostate cells led to AR tyrosine phosphorylation. In LNCaP and LAPC-4 cells, heregulin treatment induced AR tyrosine phosphorylation (Fig. $3 C$ ). To evaluate the role of Ack1 in heregulin-mediated AR phosphorylation, we tested whether Ack1 loss would suppress AR phosphorylation. Ack1 knockdown by Ack1-specific siRNA inhibited heregulin-induced AR tyrosine phosphorylation (Fig. $3 D$ ), suggesting that Ack1 was a necessary intermediary in heregulin-dependent AR phosphorylation.

Ack1 Is Required for AR-Dependent Gene Expression and Is Recruited to the Androgen-Responsive Enhancer. To examine the role of Ack1 in AR transcriptional function, the effect of knockdown of endogenous Ack1 on AR reporter activity and endogenous androgen-dependent gene expression was determined. Both androgen and heregulin stimulated the AR-dependent reporter ARR2PB-luciferase that had been transfected into LAPC-4 (Fig. 3E). Ack1 knockdown inhibited androgen- and heregulinstimulated AR reporter activity. Similarly, Ack1 knockdown inhibited androgen-stimulated expression of endogenous PSA and hK2 mRNA in both LNCaP and LAPC-4 cells (Fig. $3 G$ and $H$ and SI Fig. 10), suggesting that endogenous Ack1 was required for optimal AR function in these prostate cancer cells. Ack1 and AR knockdown had no effect on NF- $\kappa$ B-mediated induction of Bcl-3 by TNF- $\alpha$, demonstrating that the effect on androgenstimulated genes was specific (SI Fig. 10). The detection of the Ack1-AR protein complex led us to hypothesize that Ack1 may be recruited to the androgen-responsive enhancer as a component of the AR transcriptional complex. ChIP analysis was performed with antibodies specific for AR and Ack1 proteins. Androgen stimulated recruitment and binding of both $\mathrm{AR}$ and Ack1 proteins to the PSA enhancer (Fig. $3 I$ and $J$ and SI Fig. 11). Knockdown of Ack1 by siRNA decreased androgen-induced AR recruitment to the PSA enhancer, suggesting that Ack1 activity is required for the optimal androgen-regulated DNA binding of AR. ChIP analysis on the p16 gene performed as a negative control showed no recruitment of AR or Ack1 to the p16 gene promoter (data not shown). In addition to androgen, heregulin
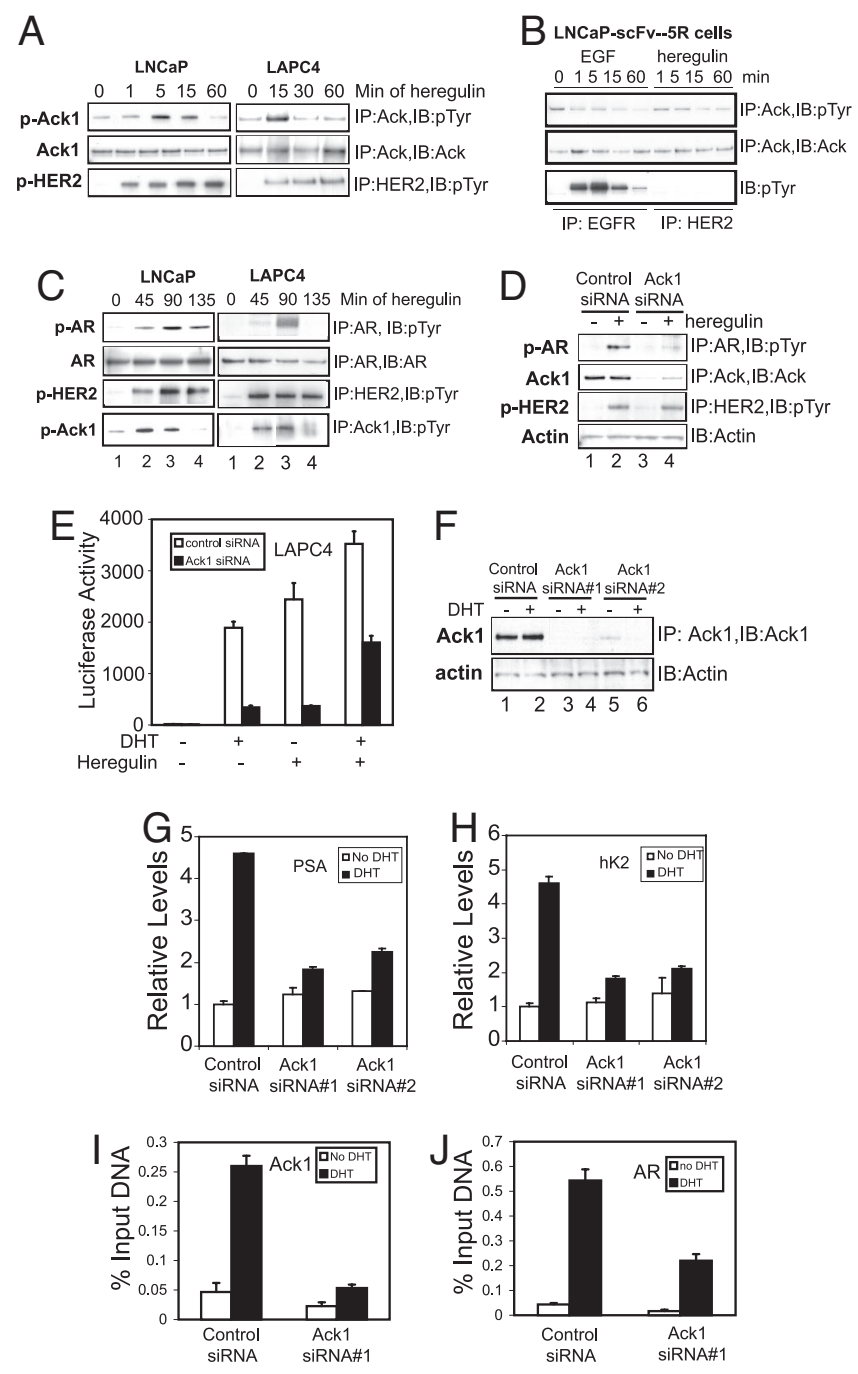

Fig. 3. Heregulin-mediated HER2 activation leads to Ack1 activation and AR tyrosine phosphorylation; Ack1 is required for AR target gene expression and recruitment. (A) LNCaP and LAPC-4 cells were treated with heregulin $(10 \mathrm{ng} / \mathrm{ml})$ for indicated times. Equal amounts of protein lysates were subjected to immunoprecipitation, followed by immunoblotting as indicated. (B) LNCaP-scFv-5R cells expressing the intracellular antibody against HER2 (14) were treated with EGF $(10 \mathrm{ng} / \mathrm{ml})$ or heregulin $(10 \mathrm{ng} / \mathrm{ml})$ for indicated time intervals, and protein lysates were subjected to immunoprecipitation followed by immunoblotting as indicated. (C) LNCaP and LAPC-4 cells were treated with heregulin $(10 \mathrm{ng} / \mathrm{ml})$ for indicated times. Equal amounts of protein lysates were subjected to immunoprecipitation, followed by immunoblotting as indicated. $(D)$ LNCaP cells were transfected with control or Ack1-specific siRNA ( $50 \mathrm{nM})$, and $48 \mathrm{~h}$ after transfection cells were treated with heregulin $(10 \mathrm{ng} / \mathrm{ml})$ for $90 \mathrm{~min}$. Equal amounts of protein lysates were subjected to immunoprecipitation, followed by immunoblotting as indicated. $(E)$ LAPC-4 cells were transfected with the ARR2PBluciferase reporter ( $500 \mathrm{ng}$ ) and the AR vector ( $50 \mathrm{ng}$ ) and control or Ack1-specific siRNA (100 nM). Twenty-four hours after transfection, cells were treated with DHT $(10 \mathrm{nM})$ or heregulin $(10 \mathrm{ng} / \mathrm{ml})$ for $16 \mathrm{~h}$, and luciferase activity was determined. $(F)$ LNCaP cells were transfected with control or Ack1-specific siRNA sequences $(50 \mathrm{nM})$. ( $G$ and $H$ ) LAPC-4 cells were transfected with control or Ack1-specific siRNA ( $50 \mathrm{nM}$ ), and $24 \mathrm{~h}$ after transfection cells were treated with DHT (10 nM) for $16 \mathrm{~h}$ or untreated. Quantitative RT-PCR for PSA $(G)$ and hK2 $(H)$ mRNA was performed. Data are representative of three similar independent experiments. ( $/$ and $J$ ) LAPC-4 cells transfected with siRNA as above were treated with DHT for $2 \mathrm{~h}$, and ChIP analysis for binding of Ack1 $(I)$ or AR $(J)$ to the ARE III enhancer of the PSA gene was performed by using quantitative PCR.

treatment of LAPC-4 cells independently stimulated recruitment and binding of both AR and Ack1 proteins to the PSA and $\mathrm{hK} 2$ enhancers, and the combination of both further enhanced 
A
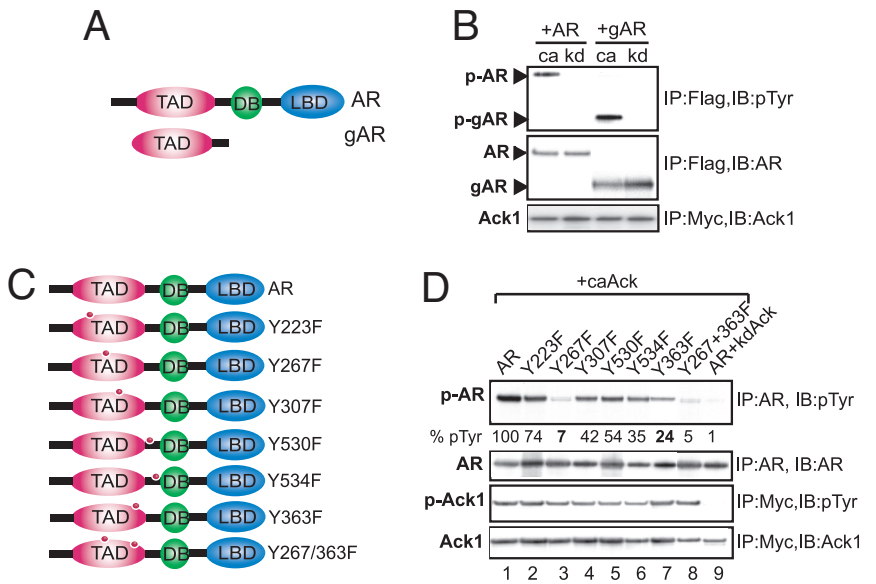

Fig. 4. Ack1 phosphorylates $A R$ at Tyr-267 and Tyr-363. (A) FLAG-tagged full-length $A R$ and the gAR-deletion construct (amino acids 142-540) are shown. (B) 293T cells were transfected with the AR or gAR expression vector $(2 \mu \mathrm{g})$, along with the caAck or kdAck expression vector $(2 \mu \mathrm{g})$. Twenty-four hours after transfection, protein lysates were subjected to immunoprecipitation, followed by immunoblotting as indicated. (C) Schematic of AR domains and point mutants. $(D) 293 T$ cells were transfected with the AR or AR point mutant expression vector ( 1 or $2 \mu \mathrm{g}$ for Y267F to keep AR protein expression levels similar) and the caAck or kdAck vector $(2 \mu \mathrm{g})$. Twenty-four hours after transfection, protein lysates were subjected to immunoprecipitation, followed by immunoblotting as indicated. Relative expression of phosphorylated AR is shown below the top panel.

recruitment of AR and Ack1 (SI Fig. 11 and data not shown). Androgen and heregulin treatment also led to increased PSA and hK2 mRNA levels, and the combination treatment further increased expression levels of these two genes (SI Fig. 11). These data suggest that Ack1 is recruited to the androgen-induced transcription complex by interacting with AR, and that Ack1 may be required for optimal AR-dependent transcription by regulating recruitment and DNA binding of AR.

Ack1 Phosphorylates AR at Tyr-267 and Tyr-363. To identify the tyrosine residue in AR targeted by Ack1, three strategies were used: $(i)$ deletion analysis using truncated AR proteins; (ii) coexpression of AR and activated Ack1 and two-step affinity purification of AR, followed by mass spectrometry; and (iii) mutational analysis of predicted AR phosphorylation sites. First, deletion analysis revealed that the gAR-deletion construct lacking carboxyl-terminal ligand- and DNA-binding domains (Fig. $4 A$ ) was effectively phosphorylated by activated Ack1 (Fig. 4B). Second, mass spectrometric analysis revealed a tryptic peptide (residues 263-288) with tyrosine phosphorylation at Tyr-267 (SI Fig. 12). The fragment ion spectra of the phosphorylated peptide in the sample are annotated to show the ions from the experimental data, which match the fragment ions from a theoretical fragmentation of the phosphorylated peptide. A site-directed mutation of Tyr-267 (Y267F) resulted in significant loss in Ack1-dependent tyrosine phosphorylation (Fig. 4D). Third, an artificial neural network-based method for predicting potential phosphorylation sites was used to search for potential tyrosine residues targeted by Ack1 (16). Four other potential tyrosine phosphorylation sites (Tyr-223, -363, -530, and -534) were identified. These point mutants and an additional point mutant $\mathrm{Y} 307 \mathrm{~F}$ as a control were generated (Fig. 4C). Activated Ack1, but not kinase-dead Ack1, phosphorylated wild-type AR in 293T cells. The Y363F mutant exhibited moderately decreased AR phosphorylation; the Y267F mutant and the double mutant Y267/363F virtually abolished Ack1-dependent AR phosphorylation (Fig. 4D).
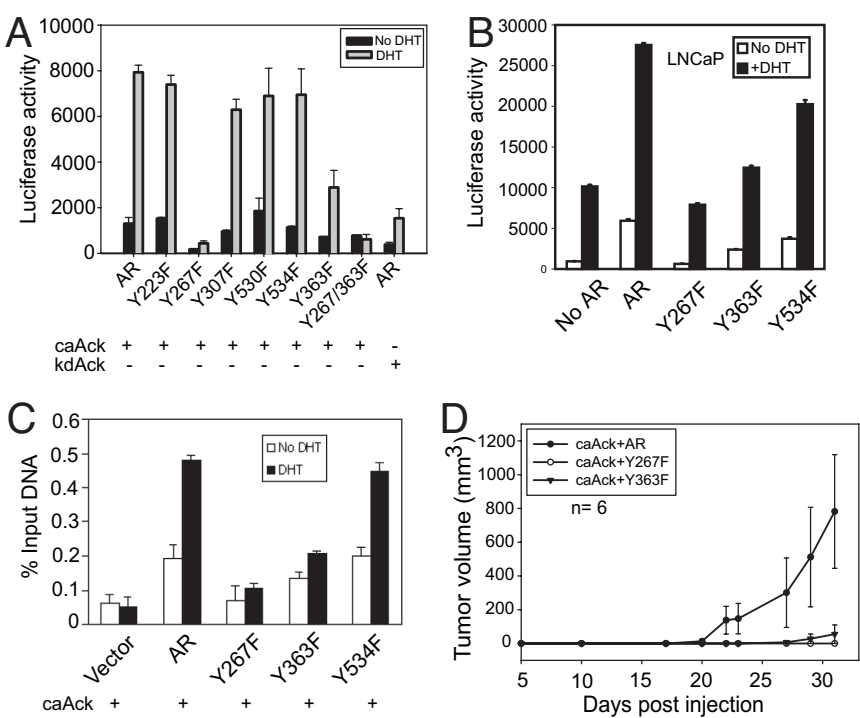

Fig. 5. Mutation of AR phosphorylation sites leads to inhibition of Ack1induced AR transactivation, DNA binding, and androgen-independent growth of xenograft tumors. (A) 293T cells were transfected with the AR or AR point mutant vector (500 ng or $1 \mu \mathrm{g}$ for Y267F), caAck (or kdAck) (500 ng), and the ARR2PB-luciferase reporter (500 ng). Twenty-four hours after transfection, cells were treated with DHT $(10 \mathrm{nM})$ for $16 \mathrm{~h}$, and luciferase activity was determined. Data shown represent mean + SE of four independent experiments. (B) LNCaP cells were transfected with ARR2PB-luciferase (500 ng) and the AR or AR point mutant vector (200 or 400 ng for Y267F) without Ack1 vector. Twenty-four hours after transfection, cells were treated with DHT (10 $\mathrm{nM}$ ) for $16 \mathrm{~h}$, and luciferase activity was determined. (C) LNCaP-caAck cells stably expressing FLAG-tagged AR or point mutants were treated with DHT for $2 \mathrm{~h}$, and ChIP analysis was performed by using FLAG antibody. Precipitated DNA was subjected to quantitative PCR analysis targeting the ARE III enhancer of the PSA gene. $(D)$ LNCaP-caAck cells stably expressing FLAG-tagged wildtype AR or Y267F or Y363F mutants of AR were injected s.c. $\left(2 \times 10^{6}\right.$ cells per injection) into the flanks of castrated nude mice.

Mutation of Ack1-Dependent AR Phosphorylation Sites Inhibits Transcriptional Activity and DNA Binding of AR and Androgen-Independent Growth of Xenograft Tumors.To probe whether Ack1-mediated AR phosphorylation was responsible for enhanced ARdependent gene expression in caAck-expressing LNCaP and LAPC4 cells, we tested the transcriptional ability of AR mutants, coexpressed with activated Ack1, by using the AR-dependent reporter ARR2PB-luciferase. Coexpression of activated Ack1 stimulated reporter activity by $\approx 8$-fold above that seen with kdAck (Fig. 5A). Y223F, Y307F, Y530F, and Y534F mutants did not significantly reduce AR transcriptional activity. In contrast, the Y363F mutant reduced activity by two-thirds, and the Y267F and the double mutant (Y267F/Y363F) virtually abolished the activated Ack1-dependent AR transcriptional stimulation in the absence or presence of androgen (Fig. $5 A$ ). Thus, Ack1-induced tyrosine phosphorylation of $\mathrm{AR}$ at two residues in the $\mathrm{AR}$ transactivation domain (Tyr-267 and Tyr-363) markedly stimulates Ack1-dependent AR transcriptional function; Tyr-267 appears to be the functionally more important site. The reporter assay in wild-type LNCaP cells (without activated Ack1) showed androgen-stimulated reporter activity due to the presence of endogenous AR. Expression of exogenous AR increased androgen-dependent reporter activity (Fig. $5 B$ ). When the Y534F mutant was expressed, reporter activity was minimally impaired, compared with the wild-type AR as previously reported (17); in contrast, the Y363F mutant was significantly compromised, and the Y267F mutant was completely inactive in this assay. These data suggest that Tyr-267 and Tyr-363 may play an important role in general AR function. 
To characterize the effect of Ack1-dependent phosphorylation sites of AR in a cellular context, FLAG-tagged AR mutants were stably expressed in LNCaP-caAck cells. ChIP analysis for androgen-induced recruitment and DNA binding to the PSA enhancer was performed by using FLAG antibody. The Y267F mutant was significantly compromised in its ability to bind to the PSA enhancer after androgen treatment; the Y363F mutant also exhibited reduced binding, but to a lesser extent than the Y267F mutant, similar to the reporter activity (Fig. 5C). Similar results were also seen for binding of these mutants to the hK2 enhancer (data not shown). Taken together, these results suggested that enhanced recruitment and DNA binding of AR induced by activated Ack1 required phosphorylation of AR at Tyr-267 and was modified by the Tyr-363 site.

To determine the functional role of AR tyrosine phosphorylation sites, LNCaP-caAck cells engineered to stably express wild-type AR or AR point mutants were implanted s.c. and their tumorigenicity was compared. LNCaP-caAck cells expressing wild-type AR formed tumors in castrated mice, whereas LNCaPcaAck cells expressing Y267F or Y363F mutants of AR did not form tumors (Fig. 5D). These data suggested that loss of phosphorylation at Tyr-267 and Tyr-363 sites in AR impaired tumor growth induced by activated Ack1, and thus the phosphorylation of AR at these sites appeared to be critically linked to the ability of activated Ack1 to promote androgenindependent growth of prostate xenograft tumors.

\section{Discussion}

Receptor tyrosine kinase HER2 has been previously shown to modulate AR signaling, in part, through its effect on the recruitment and binding of $\mathrm{AR}$ to the androgen-responsive enhancer elements $(14,15)$. Ligand-activated HER2 led to AR tyrosine phosphorylation, which was abrogated by Ack1 knockdown. Ack1 was tyrosine phosphorylated by heregulin treatment, and elimination of surface HER2 expression abolished heregulin-dependent tyrosine phosphorylation of Ack1. These results suggest that Ack1 is a critical intermediate of HER2 signaling in prostate cancer cells and its activation of ARregulated genes (13). The effect of Ack1 knockdown on androgen target gene expression such as PSA and hK2 and recruitment and DNA binding of AR is similar to the reported effect of HER2 inhibition or knockdown $(14,15)$. Cells expressing activated Ack1 exhibit enhanced recruitment and DNA binding of AR and increased androgen target gene expression. These data are consistent with the hypothesis that Ack1 is a downstream effector of HER2 on AR. Another notable finding is that Ack1 was recruited to the androgen-responsive enhancer, presumably in complex with AR, after either androgen stimulation or HER2 activation by heregulin. Heregulin-dependent AR recruitment and binding to the enhancer and induction of endogenous PSA and hK2 mRNA expression in the absence of androgen are consistent with our previous report of heregulin-dependent stimulation of AR reporter activity (13). Surprisingly, androgen alone (without heregulin) induced Ack1 recruitment to the enhancer. Ack1 recruitment into the AR transcriptional complex (or other aspects of Ack1 function) may be involved in optimal AR function because endogenous Ack1 knockdown inhibited androgen-stimulated AR reporter activity and androgen target gene expression and recruitment and DNA binding of AR (Fig. $3)$. It is possible in these prostate cancer cells that a low level of activated Ack1 exists, increasing basal AR-dependent actions. We postulate that, in addition to tyrosine phosphorylation of AR by Ack1, the presence of Ack1 in the AR transcriptional complex may stimulate AR-dependent transcription by phosphorylating other components of the AR complex.

In addition to HER2, alternate mechanisms such as other receptor tyrosine kinases (i.e., Mer) (5), amplification of the Ack1 gene (6), or kinase-activating point mutations may lead to Ack1

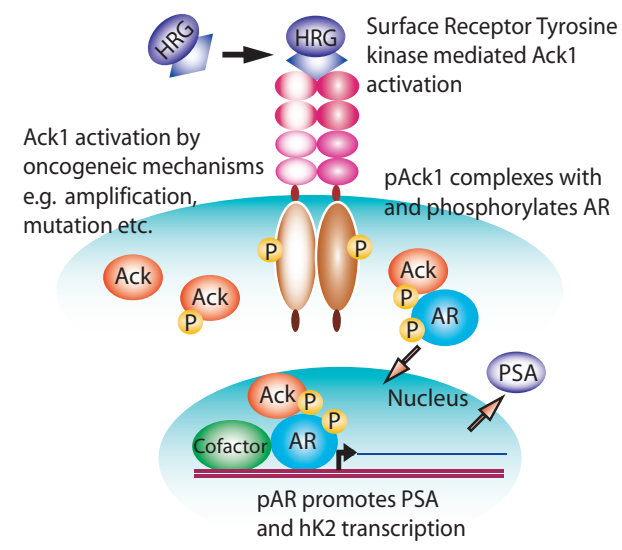

Fig. 6. Model of Ack 1 and AR activation. Cell surface receptors activated by autocrine, paracrine, or mutational events may activate the Ack 1 kinase, as indicated by Ack1 autophosphorylation. Subsequently, Ack1 binds and phosphorylates AR protein. The AR-Ack1 complex translocates to the nucleus and binds to the AREs on DNA, where it activates AR-dependent gene expression in the absence of or at suboptimal levels of androgen. Alternative mechanisms, including Ack1 gene amplification or mutation, may activate intracellular Ack1.

activation (Fig. 6). The Catalog of Somatic Mutation in Cancer database of kinase mutations in human tumors (www.sanger.ac.uk/ genetics/CGP/cosmic/) (18) shows that 5 of 210 tumor samples had point mutations in Ack1, including R99Q and E346K substitutions in the kinase domain found in ovarian cancer. Regardless of the mechanisms of activation, our results demonstrate that sustained Ack1 activation is potently tumorigenic and can change the nature of prostate cancer cells from androgen-dependent to androgenindependent. In addition to AR, there may be additional targets of Ack1. In our previous report, we identified a tumor suppressor Wwox, a WW domain containing oxidoreductase, which undergoes polyubiquitination and degradation when it is phosphorylated at Tyr-287 of Wwox by Ack1 (5). Taken together, these data indicate that Ack1 may promote tumorigenesis by targeting multiple pathways that include AR, Wwox, and perhaps other downstream Ack1 targets involved in enhanced invasion and motility of tumor cells (9). These findings identify Ack1 as an attractive target for inhibition in AICaP.

AR stimulates androgen-responsive gene expression on binding to ARE sequences on DNA; this binding is followed by the assembly of a multiprotein transcriptional complex that includes coactivators, histone acetyl transferases, and components of general transcription machinery. Ack1-dependent AR phosphorylation may regulate AR function at several steps (i.e., AR protein stability, ligand binding, nuclear translocation, DNA binding, and protein-protein interactions). The amino-terminal 485 amino acids of AR encode the activation function 1 (AF-1) transactivation domain that is predominantly responsible for transcriptional stimulatory activity of AR (19). Both Ack1dependent AR phosphorylation sites that we identified, Tyr-267 and Tyr-363, are located within this AF-1 domain. The AF-1 domain lacked stable secondary structure in aqueous solution, but may adopt a more structured and protease-resistant conformation on intra- and/or intermolecular protein-protein interactions (20). Because the N-terminal AF-1 domain has been shown to interact with the p160 family of coactivators, such as steroid receptor coactivator-1 $(21,22)$, it is possible that Ack1dependent phosphorylation sites in AR may regulate recruitment of coactivators and histone acetyl transferases, thereby regulating transcriptional activation. The mechanisms by which Ack1-dependent phosphorylation of AR regulates its activity remain to be elucidated. 
While this manuscript was in preparation, two groups reported AR phosphorylation at Tyr-534 by Src tyrosine kinase $(17,23)$. Although several kinases may be capable of phosphorylating AR, our data demonstrate that Tyr-534 is not involved in Ack1-dependent AR transactivation. Thus, it is apparent that different signaling pathways downstream of receptor tyrosine kinases mediated by nonreceptor tyrosine kinases (e.g., Ack1, $\mathrm{Src}$ ) may have distinct biochemical consequences at AR, and that AR tyrosine phosphorylation may alter its activity in a lowandrogen environment. More work will be necessary to delineate the pathophysiological role of these kinases in the progression of prostate cancer and the therapeutic effect of their inhibition.

\section{Materials and Methods}

Cells and Tumorigenicity. Pooled LNCaP cells stably expressing myc-tagged caAck, kdAck, and wild-type wAck were derived by retrovirus transduction and selection in puromycin and were implanted in nude mice (5) under the Institutional Animal Care and Use Committee-approved protocol.

Immunoprecipitation, Immunoblotting, Chromatin Immunoprecipitation, and in Vitro Kinase Analysis. LNCaP and LAPC-4 cells (24) were transfected by using Effectine (QIAGEN, Valencia, CA) or Fugene (Roche Diagnostics, Indianapolis, IN) as per the manufacturer's protocol, and immunoprecipitation and immunoblotting were performed as described earlier (5). ChIP analysis was performed by using an antibody against AR (Santa Cruz Technology, Santa Cruz, CA) or Ack1 (5) as described earlier (14). The GST-fusion protein purification and in vitro kinase assay were performed as described earlier (25). For details, see SI Methods.

1. Chen CD, Welsbie DS, Tran C, Baek SH, Chen R, Vessella R, Rosenfeld MG, Sawyers CL (2004) Nat Med 10:33-39.

2. Grossmann ME, Huang H, Tindall DJ (2001) J Natl Cancer Inst 93:1687-1697.

3. Dai B, Kim O, Xie Y, Guo Z, Xu K, Wang B, Kong X, Melamed J, Chen H, Bieberich CJ, et al. (2006) Cancer Res 66:8058-8064.

4. Graham DK, Dawson TL, Mullaney DL, Snodgrass HR, Earp HS (1994) Cell Growth Diff 5:647-657.

5. Mahajan NP, Whang YE, Mohler JL, Earp HS (2005) Cancer Res 65:1051410523.

6. van der Horst EH, Degenhardt YY, Strelow A, Slavin A, Chinn L, Orf J, Rong M, Li S, See LH, Nguyen KQ, et al. (2005) Proc Natl Acad Sci USA 102:15901-15906.

7. Mott HR, Owen D, Nietlispach D, Lowe PN, Manser E, Lim L, Laue ED (1999) Nature 399:384-388.

8. Kato J, Kaziro Y, Satoh T (2000) Biochem Biophys Res Comm 268:141-147.

9. Modzelewska K, Newman LP, Desai R, Keely PJ (2006) J Biol Chem 281:3752737535.

10. MacKeigan JP, Murphy LO, Blenis J (2005) Nat Cell Biol 7:591-600.

11. Nur-E-Kamal A, Zhang A, Keenan SM, Wang XI, Seraj J, Satoh T, Meiners S, Welsh WJ (2005) Mol Cancer Res 3:297-305.

12. Craft N, Shostak Y, Carey M, Sawyers CL (1999) Nat Med 5:280-285.

13. Gregory C, Whang Y, McCall W, Fei X, Liu Y, Ponguta L, French F, Wilson E, Earp HS (2005) Clin Cancer Res 11:1704-1712.

14. Liu Y, Majumder S, McCall W, Sartor CI, Mohler JL, Gregory CW, Earp HS, Whang YE (2005) Cancer Res 65:3404-3409.
Patient Tissue Samples. AICaP samples were obtained from transurethral resection specimens from men with urinary retention from local recurrence during androgen deprivation. $\mathrm{ADCaP}$ and BPH samples were obtained from prostatectomy specimens from men with localized prostate cancer (26). Tissue procurement and studies were performed with approval by the University of North Carolina at Chapel Hill Institutional Review Board. The frozen tumors/samples were homogenized in high-salt NLB buffer $[25 \mathrm{mmol} / \mathrm{L}$ Tris $(\mathrm{pH} 7.5), 500 \mathrm{mmol} / \mathrm{L} \mathrm{NaCl}, 1 \%$ Triton $\mathrm{X}-100,10 \%$ glycerol, $1 \mathrm{mmol} / \mathrm{L}$ EDTA, phosphatase inhibitors (10 mmol/L NaF, $1 \mathrm{mmol} / \mathrm{L} \mathrm{Na} 2 \mathrm{VO} 4)$, and protease inhibitor mix (Roche Diagnostics)].

Knockdown of Ack1 or AR by RNA Interference. The Ack1-specific siRNA (Dharmacon RNA Technologies, Lafayette, CO), ARspecific siRNA, or control siRNA were transfected by using siPORT Lipid agent (Ambion, Austin, TX) or electroporated by using Amaxa Biosystems (Gaithersburg, MD) reagents.

Reporter Assays. For details of reporter assays, see SI Methods.

We thank Natalie Edmund, Cathy Aiken, and Charlene Ross (University of North Carolina at Chapel Hill Lineberger Animal Studies Core Facility) for assistance with animal experiments; Debra Hunter for technical support; Dominic Moore for statistical analysis; Kiran Mahajan for helpful discussions; and Genentech (South San Francisco, CA) for the gift of heregulin. This work was supported by grants from Elsa U. Pardee Foundation (to N.P.M.) and National Institutes of Health Grants CA112553 (to H.S.E.), CA100700 (to Y.E.W.), CA085772 (to Y.E.W.), and CA77739 (to J.L.M.).

15. Mellinghoff IK, Vivanco I, Kwon A, Tran C, Wongvipat J, Sawyers CL (2004) Cancer Cell 6:517-527.

16. Blom N, Gammeltoft S, Brunak S (1999) J Mol Biol 294:1351-1362.

17. Guo Z, Dai B, Jiang T, Xu K, Xie Y, Kim O, Nesheiwat I, Kong X, Melamed J, Handratta VD, et al. (2006) Cancer Cell 10:309-319.

18. Forbes S, Clements J, Dawson E, Bamford S, Webb T, Dogan A, Flanagan A, Teague J, Wooster R, Futreal PA, Stratton MR (2006) Br J Cancer 94:318322.

19. Jenster G, van der Korput HA, Trapman J, Brinkmann AO (1995) J Biol Chem 270:7341-7346.

20. Reid J, Kelly SM, Watt K, Price NC, McEwan IJ (2002) J Biol Chem 277:20079-20086.

21. Alen P, Claessens F, Verhoeven G, Rombauts W, Peeters B (1999) Mol Cell Biol 19:6085-6097.

22. Bevan CL, Hoare S, Claessens F, Heery DM, Parker MG (1999) Mol Cell Biol 19:8383-8392

23. Kraus S, Gioeli D, Vomastek T, Gordon V, Weber MJ (2006) Cancer Res 66:11047-11054.

24. Klein KA, Reiter RE, Redula J, Moradi H, Zhu XL, Brothman AR, Lamb DJ, Marcelli M, Belldegrun A, Witte ON, Sawyers CL (1997) Nat Med 3:402408.

25. Mahajan NP, Earp HS (2003) J Biol Chem 278:42596-42603.

26. Titus MA, Gregory CW, Ford OH, III, Schell MJ, Maygarden SJ, Mohler JL (2005) Clin Cancer Res 11:4365-4371. 